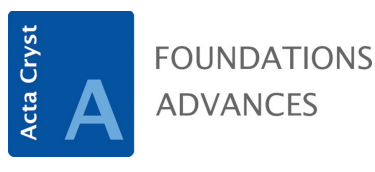

ISSN 2053-2733

Keywords: thermoelectricity; transport properties; magnetic order; galvanomagnetic effects; thermomagnetic effects

Supporting information: this article has supporting information at journals.iucr.org/a

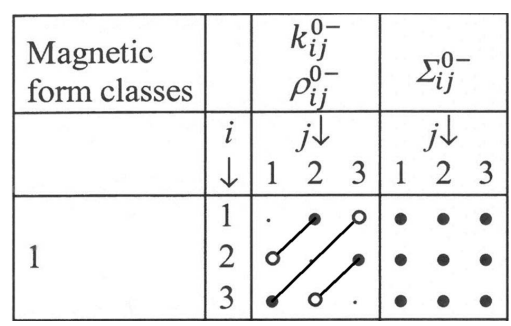

C 2020 International Union of Crystallography

\section{Thermoelectric transport properties in magnetically ordered crystals. Further corrigenda and addenda}

\author{
Hans Grimmer* \\ Multiscale Materials Experiments, Research with Neutrons and Muons, Paul Scherrer Institut, Forschungsstrasse 111, \\ Villigen PSI, CH-5232, Switzerland. *Correspondence e-mail: hans.grimmer@psi.ch
}

Further corrigenda and addenda for the article by Grimmer [Acta Cryst. (2017), A73, 333-345] are reported. New figures in the supporting information show how the restrictions on the forms of galvanomagnetic and thermomagnetic tensors are related to those on corresponding thermoelectromagnetic tensors.

Corrigenda. Corrections to Grimmer (2017) are needed in Figs. 4(c) and 6(c) for the fourth-rank tensors containing components marked in blue and in Fig. $6(b)$ for $\rho_{i j l m}^{-}, k_{i j l m}^{-}$in magnetic form class $4^{\prime}$. The corrections are included in the figures in the supporting information.

Addenda. For even tensors (invariant under space inversion $\overline{1}$ and time inversion $1^{\prime}$ ), the galvanomagnetic tensors $\rho$ and the thermomagnetic tensors $k$ are symmetric in the first two indices if the rank is even and antisymmetric if the rank is odd, whereas the thermoelectromagnetic tensors $\Sigma$ are general for all ranks. For magnetic tensors (invariant under $\overline{1}$ and changing sign under $1^{\prime}$ ), $\rho$ and $k$ are antisymmetric in the first two indices if the rank is even and symmetric if the rank is odd, whereas the tensors $\Sigma$ are general for all ranks. The intrinsic symmetry of these tensors has been discussed in more detail by Gallego et al. (2019).

Exchanging $\bullet$ and $\circ$ for certain components, new figures are obtained that clearly show how the forms of the tensors for $\rho$ and $k$ are related to those for $\Sigma$. The forms of the tensors for the limit point groups described in Grimmer (2019) are included in the supporting information in Figs. 1-6.

\section{References}

Gallego, S. V., Etxebarria, J., Elcoro, L., Tasci, E. S. \& Perez-Mato, J. M. (2019). Acta Cryst. A75, 438-447.

Grimmer, H. (2017). Acta Cryst. A73, 333-345.

Grimmer, H. (2019). Acta Cryst. A75, 409. 


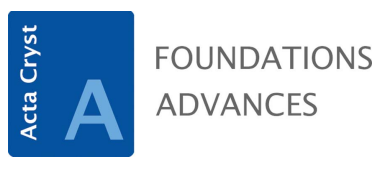

ISSN 2053-2733

Received 9 November 2018

Accepted 28 November 2018

Edited by W. F. Kuhs, Georg-August University Göttingen, Germany

Keywords: thermoelectricity; transport properties; magnetic order; galvanomagnetic effects; thermomagnetic effects

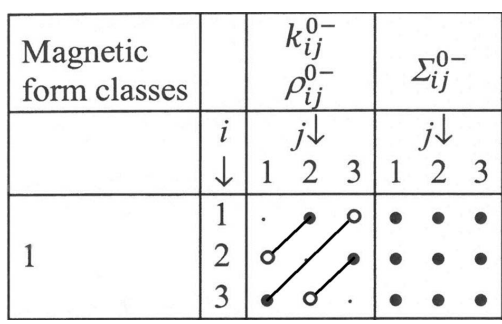

C 2019 International Union of Crystallography

\section{Thermoelectric transport properties in magnetically ordered crystals. Corrigendum and addenda}

\section{Hans Grimmer*}

Multiscale Materials Experiments, Research with Neutrons and Muons, Paul Scherrer Institut, Forschungsstrasse 111, Villigen PSI, CH-5232, Switzerland. *Correspondence e-mail: hans.grimmer@psi.ch

A correction and additions concerning the limiting point groups are made to the article by Grimmer [Acta Cryst. (2017), A73, 333-345].

Corrigendum: Restriction ** in Fig. 4(c) of Grimmer (2017) should be $\Sigma_{1212}^{-}=\frac{1}{2}\left(\Sigma_{1111}^{-}-\Sigma_{1122}^{-}\right)$.

Addenda: If polycrystalline materials are considered instead of single crystals, it is often presumed that their symmetry can be described by a limit point group (Curie group). A recent example is given by Uchida et al. (2018). They measured the anisotropy of the quadratic magnetoPeltier effect in ferromagnetic polycrystalline nickel, presuming the material to be isotropic. It is therefore useful to consider, in addition to the crystallographic space-time point groups, the limit continuous ones. This leads to the following additions to Table 3 in Grimmer (2017):

\begin{tabular}{lll}
\hline Category & Magnetic form class & Point groups \\
\hline II & $\infty$ & $\infty, \infty / m$ \\
& $\infty 2$ & $\infty 2, \infty m, \infty / m m$ \\
III $^{\text {a1 }}$ & $\infty \infty$ & $\infty \infty, \infty \infty m$ \\
\hline
\end{tabular}

Add in the first column of Fig. 1 ' $\infty 1$ ' ' in the third cell from the bottom, ' $\infty 21$ ' in the second cell from the bottom and ' $\infty \infty 1^{\prime \prime}$ in the bottom cell.

Add in the bottom cell of the first column of Fig. 2(b) ' $\infty \infty 1^{\prime}$ ' and '*'.

Add below Fig. 2(b) '* In Laue class $\infty \infty 1^{\prime}$ the components of the fourth-rank tensor satisfy the restriction $R_{1212}=\frac{1}{2}\left(R_{1111}\right.$ $-R_{1122}$ ), where $R$ stands for $\rho^{+}, k^{+}$and $\Sigma^{+}$.

Add in the first column of Fig. $2(c)$ ' $\infty 1^{\prime}$ ' in the second cell from the bottom and ' $\infty 21^{\prime}$ ' in the bottom cell.

Add in the first column of Fig. 3 ' $\infty$ ' in the third cell from the bottom, ' $\infty 2$ ' in the second cell from the bottom and ' $\infty \infty$ ' in the bottom cell.

Add in the bottom cell of the first column of Fig. 4(b) ' $\infty \infty$ ' and ' $*$ '.

Add below Fig. 4(b) ** In magnetic form class $\infty \infty$ the components of the fourth-rank tensor satisfy the restriction $\Sigma_{1212}^{-}=\frac{1}{2}\left(\Sigma_{1111}^{-}-\Sigma_{1122}^{-}\right)$.

Add in the first column of Fig. 4(c) ' $\infty$ ' in the second cell from the bottom and ' $\infty 2$ ' in the bottom cell.

Add in the first column of Fig. 5 ' $\infty 2$ ' in the second cell from the bottom.

Add in the first column of Fig. $6(c)$ ' $\infty 2$ ' ' in the fourth cell from the bottom.

\section{References}

Grimmer, H. (2017). Acta Cryst. A73, 333-345.

Uchida, K. I., Daimon, S., Iguchi, R. \& Saitoh, E. (2018). Nature, 558, 95-99. 